Research Article

\title{
Prediction of Continuous Rain Disaster in Henan Province Based on Markov Model
}

\author{
Xiaoxiao Zhu (D), Shuhua Zhang, and Bingjun Li (iD) \\ College of Information and Management Science, Henan Agricultural University, Zhengzhou 450002, China \\ Correspondence should be addressed to Bingjun Li; zzlbjun@163.com
}

Received 24 July 2019; Revised 21 February 2020; Accepted 27 February 2020; Published 12 September 2020

Academic Editor: Seenith Sivasundaram

Copyright (C) 2020 Xiaoxiao Zhu et al. This is an open access article distributed under the Creative Commons Attribution License, which permits unrestricted use, distribution, and reproduction in any medium, provided the original work is properly cited.

Continuous rain disasters occur frequently, which seriously affect maize yield. However, the research on predicting continuous rain disasters is very limited. Taking the maize in Henan Province as an example, the Markov model is used to predict the occurrence of continuous rain in the middle growth and late growth stages (flowering and filling stages) of 13 cities in Henan Province. The results showed that the maize in Henan Province would suffer from continuous rain disaster in 2020 and 2021. Finally, combined with the prediction results, policy recommendations for maize growth in Henan Province are proposed to ensure stable and high yield of maize.

\section{Introduction}

Henan Province is an important major grain-producing area in China. Maize is the main grain crop in Henan Province. Summer maize-planting area and production account for about $10 \%$ of the country, and its output is high but unstable. During the growing period of summer maize (June to September), it is highly susceptible to various natural disasters (droughts, floods, heavy winds, and continuous rain). Among them, the early summer drought, critical drought, and rain in the flowering period have the greatest impact. Studying the effects of precipitation of summer maize from the flowering to filling stage on maize yield is of great significance for the stable yield and high yield of summer maize.

Maize is a kind of short-day C4 plant which likes warm, light, and high light effects [1]. Long-term continuous rains have severely affected maize yield. In addition, insufficient flowering during the flowering period has led to the largest reduction in yield [2]. Continuous rainy weather causes harm to agricultural production, mainly because the prolonged rainy weather will cause waterlogging in crops due to excessive moisture. In addition, due to severely insufficient light and low temperatures, it is easy to cause rotten seeds and seedling rot. Autumn crops are in the stage of filling and maturity, and frequent occurrence of rain will seriously affect the yield and quality of crops.

Scholars at home and abroad have conducted a lot of research on the relationship between continuous rain and crop growth. Based on the statistical data of tasseling to silking period of summer maize in Henan Province, Cheng and Liu [3] constructed the risk intensity sequence of continuous rainy weather to carry out the zoning of continuous rainy during summer maize flowering period in the whole province. Dietzel et al. [4] used the Agricultural Production Systems sIMulator (APSIM), based on the measurement data of maize and soybean planting system in central Iowa, the United States, to explore and analyze the impact of drought and continuous rain on the yield of corn and soybean. The analysis showed that in this area, $430 \mathrm{~mm}$ seasonal rainfall was the best water use efficiency of corn, and the best water use efficiency of soybean was $317 \mathrm{~mm}$. It provides a framework and basis for effective water use in planting system. Zhao et al. [5] analyzed the effects of spring cold continuous rain on rice, wheat, rape, and tea in Xinyang City. Zhang et al. [6] analyzed the climate change characteristics, mutations, and periodic changes 
of the number of consecutive rains in Ningxia during the whole year and the flood season (May to September). Zhang et al. [7] analyzed the annual change trend of continuous rain in spring, summer, and autumn in southwest Shandong Province. And the effects of continuous rain on crop growth, yield, and autumn harvest were also analyzed. Li and Guo [8] divided flowering rainy risk zones of summer maize in Henan Province based on satellite remote sensing and GIS. Wang and Zhang [9] and Wang [10] analyzed the impact of continuous rain disaster on maize production in Hunan Province, Nenjiang County of Heilongjiang Province and proposed corresponding preventive measures. Liu [11] adopted the objective analysis method to conduct a comprehensive analysis of the continuous rainy weather in Henan Province in September 2011.

Some of the scholars analyzed the characteristics of continuous rain occurrence, the influence of continuous rain on the growth of maize, or the division of rainy risk areas in the flowering period from a qualitative perspective. In addition, previous studies by scholars tend to make policy suggestions based on the analysis of the current situation, and there are few predictions of continuous rain disasters. In fact, it is extremely urgent to predict the continuous rain disaster. Meteorological data are big data, and there are few available data in big data. First, Markov is used to predict the continuous rainy disaster in the middle and late stages of maize growth in 13 cities of Henan Province. The results of the method are consistent with the actual conditions. Finally, based on the prediction results, policy recommendations for maize production in Henan Province are proposed.

\section{Markov Model}

The research object of the Markov Probability Matrix Prediction model is a stochastic dynamic system. It predicts the development of the system based on the probability of transition between states. The Markov Probability Matrix is suitable for predicting data columns with large random fluctuations [12].

The Markov forecasting model [13] is as follows:

First, divide the state of the prediction object $\otimes_{i}=\left[a_{i}, b_{i}\right], i=1,2, \ldots, s . \quad$ Let $\quad P_{i j}^{(k)}=P\left(X_{m+k}=j \mid\right.$ $\left.X_{m}=i\right),(i, j) \in I$, which denote the probability that the system is in the state $j$ at time $m+k$ under the condition that the system in the state $i$ at the moment $m$. That is, the transition probability of the $k$ step is experienced; by sequentially sorting $P_{i j}^{(k)}$, the following matrix can be obtained:

$$
P^{(k)}=\left[\begin{array}{cccc}
P_{11}^{(k)} & P_{12}^{(k)} & \cdots & P_{1 n}^{(k)} \\
P_{21}^{(k)} & P_{22}^{(k)} & \cdots & P_{2 n}^{(k)} \\
\vdots & \vdots & & \vdots \\
P_{n 1}^{(k)} & P_{n 2}^{(k)} & \cdots & P_{n n}^{(k)}
\end{array}\right] .
$$

Then, the matrix is the $k$-step transition probability matrix of Markov chain. Here, $\sum_{j=1}^{n} P_{i j}^{(k)}=1$.
If $M_{i j}(k)$ is the number of original data samples transferred from state $\otimes_{i}$ to state $\otimes_{j}$ through $k$ steps and $M_{i}$ is the number of original data samples in state $\otimes_{i}$, then call

$$
P_{i j}^{(k)}=\frac{M_{i j}(k)}{M_{i}}, \quad i, j=1,2, \ldots, n,
$$

the state transition probability. Since the final state of the data sequence is not clear, the last $k$ data in the data sequence should be removed when calculating $M_{i}$.

\section{Collection and Collation of Data}

According to the meteorological disaster criteria of Cheng and Liu [14] for continuous overcast rain in the flowering period, precipitation (daily precipitation $0.1 \mathrm{~mm}$ ) in continuous overcast rain for 3 consecutive days or above is regarded as a continuous rainy process. In the continuous rainy period of above 3 days, no precipitation is allowed for 1 day, but the sunshine duration should be less than 2 hours. During the process of continuous rain, a small amount of precipitation is allowed, but the sunshine duration should be less than 4 hours. At the same time, it is stipulated that each region $\geq 2 / 3$ stations appear a continuous rain process in a year, which is called a continuous rain year. The effect of continuous rain on summer maize in the middle and late stages are the most serious. The data of flowering stage and filling stage of summer maize in Henan Province were analyzed in this paper. The flowering stage is chosen from the beginning of the tasseling period to the general stage of silking, and the filling stage is chosen from the general stage of silking to the general stage of milk maturing. The statistical period of meteorological data of flowering period is July 23 to August 8, and that of the filling period is August 9 to August 30.

Agricultural meteorological observation data of summer maize in Henan Province from 2013 to 2019 from China Meteorological Data Service Center [15]. Among them, the selected meteorological indicators include daily precipitation $(0.1 \mathrm{~mm})$ and sunshine duration $(\mathrm{h})$. Considering the comprehensiveness of variable index selection, the availability of data, and the scientific rigor of data calculation, this paper selects the meteorological data of summer maize from 13 representative cities in the five regions of eastern Henan, western Henan, central Henan, southern Henan, and northern Henan for analysis and prediction. The corresponding 13 weather stations are Anyang City (53898), Xinxiang City (53986), Sanmenxia City (57051), Luoyang City, Zhengzhou City (57083), Xuchang City (57089), Kaifeng City (57091), Nanyang City (57178), Pingdingshan City, Zhoukou City, Zhumadian City (57290), Xinyang City (57297), and Shangqiu City (58005). The observations of meteorological data of Luoyang City, Pingdingshan City, and Zhoukou City were replaced by observation data of Mengjin county (57071), Baofeng county (57181), and Xihua county (57193) meteorological stations.

On the basis of the data collected by the China Meteorological Data Service Center and in accordance with the classification criteria of continuous rain disaster, the occurrence of continuous rain disaster in 13 cities of Henan Province 
is obtained as shown in Table 1. Among them, the occurrence of meteorological disaster is defined as 1 and does not occur as 0 .

According to the classification criteria of continuous rainy years, it can be determined that 2014, 2016, 2017, 2018, and 2019 are continuous rainy years in Henan province. Therefore, the continuous rainy years in Henan Province can be predicted.

\section{Result Analysis}

4.1. Markov Prediction Result Analysis. We determine the state transition probability matrix mainly by the number of occurrences of continuous rain disaster. Taking Zhengzhou, Henan Province as an example, it is set that there is no continuous rain disaster as $M_{1}$, and that of continuous rain disaster is $M_{2}$. So we get

$$
\begin{aligned}
& M_{11}(1)=2, \\
& M_{12}(1)=3, \\
& M_{21}(1)=3, \\
& M_{22}(1)=5 .
\end{aligned}
$$

State transition probability is

$$
\begin{aligned}
& p_{11}^{(1)}=\frac{3}{5} \\
& p_{12}^{(1)}=\frac{2}{5}, \\
& p_{21}^{(1)}=\frac{3}{8} \\
& p_{22}^{(1)}=\frac{5}{8} .
\end{aligned}
$$

The state transition probability matrix is

$$
P_{1}^{(1)}=\left[\begin{array}{cc}
\frac{3}{5} & \frac{2}{5} \\
\frac{3}{8} & \frac{5}{8}
\end{array}\right] \text {. }
$$

Since Zhengzhou City has not experienced continuous rain during the filling period in 2019, according to the maximum probability criterion, we can get

$$
\max \left\{p_{11}^{(1)}, p_{12}^{(1)}\right\}=p_{11}^{(1)}=\frac{5}{8}
$$

Therefore, it can be predicted that Zhengzhou City maize in 2020 may not suffer from continuous rain disaster at the flowering stage.

Similarly, the state transition probability matrices of Anyang City $P_{2}^{(1)}$, Xinxiang City $P_{3}^{(1)}$, Sanmenxia City $P_{4}^{(1)}$, Luoyang City $P_{5}^{(1)}$, Xuchang City $P_{6}^{(1)}$, Kaifeng City $P_{7}^{(1)}$, Nanyang City $P_{8}^{(1)}$, Pingdingshan City $P_{9}^{(1)}$, Zhoukou City $P_{10}^{(1)}$, Zhumadian City $P_{11}^{(1)}$, Xinyang City $P_{12}^{(1)}$, and Shangqiu City $P_{13}^{(1)}$ are as follows:

$$
\begin{aligned}
& P_{2}^{(1)}=\left[\begin{array}{ll}
\frac{1}{5} & \frac{4}{5} \\
\frac{3}{8} & \frac{5}{8}
\end{array}\right], \\
& P_{3}^{(1)}=\left[\begin{array}{ll}
\frac{1}{2} & \frac{1}{2} \\
\frac{3}{7} & \frac{4}{7}
\end{array}\right],
\end{aligned}
$$

$$
P_{4}^{(1)}=\left[\begin{array}{cc}
\frac{1}{4} & \frac{3}{4} \\
\frac{1}{3} & \frac{2}{3}
\end{array}\right],
$$$$
P_{5}^{(1)}=\left[\begin{array}{ll}
\frac{1}{3} & \frac{2}{3} \\
\frac{4}{7} & \frac{3}{7}
\end{array}\right] \text {, }
$$$$
P_{6}^{(1)}=\left[\begin{array}{ll}
\frac{1}{5} & \frac{4}{5} \\
\frac{5}{8} & \frac{3}{8}
\end{array}\right] \text {, }
$$$$
P_{7}^{(1)}=\left[\begin{array}{cc}
\frac{1}{5} & \frac{4}{5} \\
\frac{3}{8} & \frac{5}{8}
\end{array}\right] \text {, }
$$$$
P_{8}^{(1)}=\left[\begin{array}{ll}
\frac{1}{2} & \frac{1}{2} \\
\frac{3}{7} & \frac{4}{7}
\end{array}\right] \text {, }
$$$$
P_{9}^{(1)}=\left[\begin{array}{ll}
\frac{4}{7} & \frac{3}{7} \\
\frac{2}{3} & \frac{1}{3}
\end{array}\right] \text {, }
$$$$
P_{10}^{(1)}=\left[\begin{array}{cc}
\frac{3}{5} & \frac{2}{5} \\
\frac{3}{8} & \frac{5}{8}
\end{array}\right] \text {, }
$$

$$
P_{11}^{(1)}=\left[\begin{array}{cc}
0 & 1 \\
\frac{2}{11} & \frac{9}{11}
\end{array}\right],
$$

$$
P_{12}^{(1)}=\left[\begin{array}{ll}
\frac{1}{5} & \frac{4}{5} \\
\frac{3}{8} & \frac{5}{8}
\end{array}\right] \text {, }
$$

$$
P_{13}^{(1)}=\left[\begin{array}{ll}
\frac{1}{2} & \frac{1}{2} \\
\frac{4}{7} & \frac{3}{7}
\end{array}\right] \text {. }
$$


TABLE 1: The occurrence of continuous rain disasters in 13 cities of Henan province.

\begin{tabular}{|c|c|c|c|c|c|c|c|c|}
\hline City & Date & 2013 & 2014 & 2015 & 2016 & 2017 & 2018 & 2019 \\
\hline \multirow{2}{*}{ Anyang } & $7.29-8.8$ & 0 & 1 & 1 & 1 & 1 & 0 & 1 \\
\hline & $8.9-8.30$ & 0 & 1 & 0 & 0 & 1 & 1 & 1 \\
\hline \multirow{2}{*}{ Xinxiang } & $7.29-8.8$ & 0 & 1 & 1 & 1 & 1 & 1 & 0 \\
\hline & $8.9-8.30$ & 0 & 1 & 1 & 0 & 0 & 0 & 0 \\
\hline \multirow{2}{*}{ Sanmenxia } & $7.29-8.8$ & 0 & 1 & 1 & 1 & 1 & 0 & 1 \\
\hline & $8.9-8.30$ & 0 & 1 & 1 & 0 & 1 & 1 & 0 \\
\hline \multirow{2}{*}{ Luoyang } & $7.29-8.8$ & 0 & 1 & 1 & 1 & 1 & 0 & 1 \\
\hline & $8.9-8.30$ & 0 & 1 & 0 & 0 & 0 & 1 & 0 \\
\hline \multirow{2}{*}{ Zhengzhou } & $7.29-8.8$ & 1 & 1 & 1 & 1 & 0 & 1 & 1 \\
\hline & $8.9-8.30$ & 0 & 1 & 1 & 0 & 0 & 0 & 1 \\
\hline \multirow{2}{*}{ Xuchang } & $7.29-8.8$ & 1 & 1 & 0 & 1 & 1 & 0 & 1 \\
\hline & $8.9-8.30$ & 0 & 1 & 0 & 0 & 1 & 1 & 0 \\
\hline \multirow{2}{*}{ Kaifeng } & $7.29-8.8$ & 0 & 1 & 1 & 1 & 1 & 1 & 1 \\
\hline & $8.9-8.30$ & 0 & 0 & 0 & 0 & 1 & 1 & 1 \\
\hline \multirow{2}{*}{ Nanyang } & $7.29-8.8$ & 0 & 1 & 0 & 1 & 0 & 1 & 0 \\
\hline & $8.9-8.30$ & 1 & 1 & 1 & 0 & 0 & 1 & 0 \\
\hline \multirow{2}{*}{ Pingdingshan } & $7.29-8.8$ & 1 & 1 & 0 & 1 & 1 & 0 & 1 \\
\hline & $8.9-8.30$ & 0 & 0 & 0 & 1 & 0 & 0 & 0 \\
\hline \multirow{2}{*}{ Zhoukou } & $7.29-8.8$ & 1 & 1 & 0 & 0 & 1 & 1 & 1 \\
\hline & $8.9-8.30$ & 1 & 1 & 0 & 0 & 0 & 1 & 0 \\
\hline \multirow{2}{*}{ Zhumadian } & $7.29-8.8$ & 1 & 1 & 0 & 1 & 1 & 1 & 1 \\
\hline & $8.9-8.30$ & 1 & 1 & 1 & 0 & 1 & 1 & 1 \\
\hline \multirow{2}{*}{ Xinyang } & $7.29-8.8$ & 0 & 1 & 0 & 1 & 1 & 0 & 1 \\
\hline & $8.9-8.30$ & 1 & 1 & 0 & 0 & 1 & 1 & 1 \\
\hline \multirow{2}{*}{ Shangqiu } & $7.29-8.8$ & 1 & 0 & 0 & 1 & 1 & 1 & 1 \\
\hline & $8.9-8.30$ & 1 & 0 & 0 & 0 & 0 & 1 & 0 \\
\hline
\end{tabular}

By observing the results predicted by Markov, it can be seen that the 7 cities of Anyang City, Sanmenxia City, Luoyang City, Xuchang City, Kaifeng City, Zhumadian City, and Xinyang City may suffer from continuous rain disaster at the flowering stage in 2020. However, 2 cities of Pingdingshan City and Zhoukou City may not suffer from continuous rain disaster at the flowering stage in 2020. However, we cannot determine whether maize in Xinxiang City, Nanyang City, and Shangqiu City suffers from continuous rain disaster at the flowering stage in 2020 .

Based on the map of Henan Province (Figure 1), it can be seen that Xinxiang City borders Anyang City, and the maize in Anyang City may suffer from continuous rain disaster at the flowering stage in 2020. Therefore, it is predicted that the maize in Xinxiang City may suffer from continuous rain disaster at the flowering stage in 2020. Nanyang City is surrounded by Sanmenxia City, Luoyang City, Pingdingshan City, Zhumadian City, and Xinyang City. Except for Pingdingshan City, the remaining 4 cities may suffer from continuous rain disaster at the flowering stage in 2020. Therefore, it can be concluded that the maize in Nanyang City may suffer from continuous rain disaster at the flowering stage in 2020. Shangqiu City borders Kaifeng City and Zhoukou City. Kaifeng City may suffer from continuous rain disaster at the flowering stage in 2020, while Zhoukou City may not suffer from continuous rain disaster at the flowering stage in 2020. As we can see from the map,

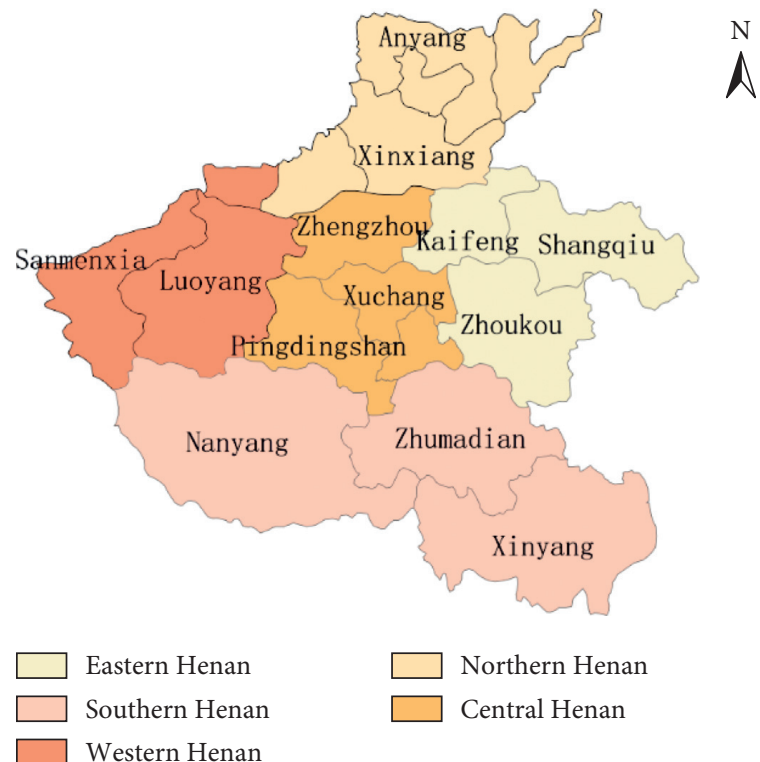

Figure 1: Map of 13 cities in Henan province.

Shangqiu City and Zhoukou City have more borders and tend to be in the direction of Zhoukou City. Therefore, it is predicted that the maize in Shangqiu City will not suffer from continuous rain disaster at the flowering stage in 2020. In the same way, the situation of the maize in 13 cities of Henan Province is it suffers from continuous rain disaster in 2020 
TABle 2: Prediction of the occurrence of continuous rain disasters in 13 cities of Henan Province.

\begin{tabular}{lcccc}
\hline & \multicolumn{2}{c}{2020} & \multicolumn{2}{c}{2021} \\
\hline Date & $7.29-8.8$ & $8.9-8.30$ & $7.29-8.8$ & $8.9-8.30$ \\
Anyang & 1 & 1 & 1 & 1 \\
Xinxiang & 1 & 1 & 1 & 1 \\
Sanmenxia & 1 & 1 & 1 & 1 \\
Luoyang & 1 & 0 & 1 & 0 \\
Zhengzhou & 1 & 1 & 1 & 0 \\
Xuchang & 1 & 0 & 1 & 0 \\
Kaifeng & 1 & 1 & 1 & 1 \\
Nanyang & 1 & 1 & 1 & 0 \\
Pingdingshan & 0 & 0 & 0 & 0 \\
Zhoukou & 0 & 0 & 0 & 0 \\
Zhumadian & 1 & 1 & 1 & 1 \\
Xinyang & 1 & 1 & 1 & 1 \\
Shangqiu & 0 & 0 & 0 & 0 \\
\hline
\end{tabular}

flowering period and 2020 filling period and 2021 flowering period and 2021 filling period, as shown in Table 2.

It is found that maize in Henan Province will suffer from large-scale continuous rain disasters in 2020 and 2021, and it will be rainy years in 2020 and 2021.

\section{Conclusion}

The continuous rain weather process affects the growth and development of maize and then affects the yield of maize. Based on the Markov model for predicting the continuous rainy weather in the middle and late stages of maize growth in 13 cities of Henan Province, the main conclusions are as follows:

(1) Research results: it is predicted that Henan Province will be rainy years in 2020 and 2021. The maize in Henan Province will suffer continuous rain disasters on a large scale. All cities should take precautions in advance to avoid the impact of continuous rain on maize production.

(2) The main effects of continuous rain on maize growth are as follows: less sunshine, insufficient light, which is not conducive to photosynthesis, and the growth of maize is weak. The grain weight of maize decreased due to the decrease in grain filling intensity. Due to the high humidity, the maize will be mildewed and budded and the color of the maize will not be bright. The temperature is too low, the accumulated temperature of the maize is insufficient, and it is prone to pests and diseases. The grain filling decreased, which results in the delay of maize maturity.

(3) The main prevention of continuous rain disaster is as follows: timely trench drainage, middle ploughing loose soil, in order to increase soil permeability, reducing farmland soil moisture, and preventing waterlogging (damage). The effects of continuous rain disaster can be alleviated to a certain extent by selecting waterlogging resistant and lodging resistant varieties. Supporting irrigation, drainage, fertilizer, pest control, meteorological forecasting, and other facilities and scientific research institutions. Preparations should be carried out for farmland capital construction in advance. At the same time, the government should increase insurance support for maize producers [9], so that maize producers can be protected from various risks such as continuous rain disaster weather, ensuring stable production. The government can reasonably organize and distribute the resources such as labor, capital, technology, agricultural machinery, tools, information, and insurance in the production of peasant households through taking certain management means and measures. In order to reduce waste, realize the purpose of optimal allocation and combination of resources, and promote agricultural production bumper harvest.

\section{Data Availability}

Agricultural meteorological observation data of summer maize in Henan Province from 2011 to 2017 from "China Meteorological Data Service Center" presented in this manuscript are open and available.

\section{Conflicts of Interest}

The authors declare that they have no conflicts of interest.

\section{Authors' Contributions}

Bingjun Li was responsible for proposing the overall idea and framework of the manuscript. Xiaoxiao Zhu was responsible for data processing and writing of the first draft of the manuscript. Shuhua Zhang was responsible for data collection and made the revision of the manuscript.

\section{Acknowledgments}

This work was supported by the Key Project of Soft Science Research in Henan Province (202400410051).

\section{References}

[1] W. D. Yu and H. L. Chen, "Study on precise comprehensive agricultural climate regional planning of summer maize in henan province," Meteorological and Environmental Sciences, vol. 33, no. 2, pp. 14-19, 2010.

[2] A. Cerrudo, J. Di Matteo, E. Fernandez, M. Robles, L. O. Pico, and F. H. Andrade, "Yield components of maize as affected by short shading periods and thinning," Crop and Pasture Science, vol. 64, no. 6, pp. 580-587, 2013.

[3] L. Cheng and R. H. Liu, "Disaster index of continuous overcast and rainy day during mid and late growth period of summer maize," Chinese Journal of Agrometeorology, vol. 35, no. 2, pp. 221-227, 2014.

[4] R. Dietzel, M. Liebman, R. Ewing et al., "How efficiently do corn-and soybean-based cropping systems use water? A systems modeling analysis," Glob Change Biol, vol. 22, pp. 666-681, 2016. 
[5] H. Zhao, Y. Wang, G. Li et al., "Method for estimating impact of spring low-temperature and continuous-rain disasters on crop yields," Meteorological Science and Technology, vol. 39, no. 1, pp. 102-105, 2011.

[6] Z. Zhang, P. Liang, Y. H. Chen et al., "Study on the characteristics of climatic changes of continuous rain in Ningxia," Journal of Catastrophology, vol. 25, no. 1, pp. 69-72, 2010.

[7] C. Y. Zhang, J. H. Fan, and X. Feng, "Law of continuous prediction occurrence and its influence on autumn harvesting and planting in southwestern shandong province," Journal of China Agricultural Resources and Regional Planning, vol. 37, no. 4, pp. 142-146, 2016.

[8] J. L. Li and Q. L. Guo, "Risk zoning method of flowering phase continuous rain of summer maize based on remote sensing and GIS," Remote Sensing Information, vol. 30, no. 2, pp. 77-79, 2015.

[9] Y. Wang and L. Zhang, "Analysis of the influence of low temperature and continuous rain on maize in hunan province," South China Agriculture, vol. 10, no. 21, pp. 221-223, 2016.

[10] J. Wang, "Analysis of climatic conditions for maize planting in Nenjiang county, Heilongjiang province," Beijing Agriculture, vol. 34, pp. 134-135, 2015.

[11] J. Liu, "Characteristic analysis of continuous rainy weather in september 2011 in henan," Meteorological and Environmental Sciences, vol. 35, no. 4, pp. 22-26, 2012.

[12] Y. K. Liu, C. L. Xie, Z. J. Yu, and S. H. Ling, "Trend prediction methods study of nuclear power plant based on GM $(1,1)$ and grey markov GM $(1,1)$ models," Atomic Energy Science and Technology, vol. 45, no. 9, pp. 1075-1079, 2011.

[13] Q. Y. Zhang, X. H. Zhou, and W. T. Wang, "The forecast for the wear trend of the diesel engine based on grey Markov chain model," Lubrication Engineering, vol. 09, pp. 145-147, 2007.

[14] L. Cheng and R. H. Liu, "Disaster risk zoning of continuous rain during florescence of summer maize in Henan Province, Central China," Chinese Journal of Ecology, vol. 31, no. 12, pp. 3075-3079, 2012.

[15] China Meteorological Data Service Center, http://data.cma. $\mathrm{cn} /$ data/cdcindex/cid/0b9164954813c573.html. 